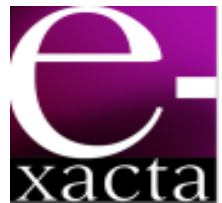

ISSN: 1984-3151

\title{
PREDIÇÃO DO COEFICIENTE DE EXPANSÃO TÉRMICA E DA ENERGIA DE ATIVAÇÃO DO ÓLEO BRUTO DE MAMONA ATRAVÉS DA APLICAÇÃO DA ANÁLISE DE REGRESSÃO LINEAR
}

\author{
PREDICTION OF THE THERMAL EXPANSION COEFFICIENT AND THE \\ ACTIVATION ENERGY OF THE CRUDE CASTOR OIL THROUGH THE \\ APPLICATION OF LINEAR REGRESSION ANALYSIS
}

\author{
César Augusto Canciam ${ }^{1}$
}

\begin{abstract}
Mestre em Engenharia Química. UEM. 2001. Professor da Universidade Tecnológica Federal do Paraná - Campus Ponta Grossa, PR. canciam@utfpr.edu.br
\end{abstract}

Recebido em: 09/09/2011 - Aprovado em: 01/11/2011 - Disponibilizado em: 30/12/2011

\begin{abstract}
RESUMO: O óleo de mamona (Ricinus communis L.) apresenta várias aplicações na área industrial, com perspectiva de utilização como fonte energética na produção de biocombustível. O objetivo deste trabalho foi predizer o coeficiente de expansão térmica e a energia de ativação do óleo bruto de mamona das cultivares BRS149 Nordestina e BRS-188 Paraguaçu, a partir de dados experimentais de outros trabalhos. Através da análise de regressão linear da densidade em função da temperatura foi possível predizer o coeficiente de expansão térmica para as cultivares. Com relação à viscosidade, através da análise de regressão linear, foi possível predizer a energia de ativação de cada cultivar. Para as cultivares BRS-149 Nordestina e BRS-188 Paraguaçu, os coeficientes de expansão térmica foram iguais, respectivamente, a 5,2745 x $10^{-4}{ }^{\circ} \mathrm{C}^{-1}$ e $5,2705 \times 10^{-4}{ }^{\circ} \mathrm{C}^{-1}$, enquanto que as energias de ativação foram iguais a $51,1468 \mathrm{~kJ}^{\mathrm{mol}}{ }^{-1}$ e $52,1686 \mathrm{~kJ} \mathrm{~mol}^{-1}$. Em todos os casos, o coeficiente de correlação foi superior a 0,99.
\end{abstract}

PALAVRAS-CHAVE: Expansão. Energia de ativação. Óleo bruto de mamona.

ABSTRACT: The castor oil (Ricinus communis L.) presents several applications in the industrial area, with perspective as energy source in the biofuel production. The objective of this work was to predict the thermal expansion coefficient and the activation energy of the crude castor oil of two cultivates BRS-149 Nordestina and BRS-188 Paraguaçu, starting from experimental data of other works. Through the analysis of lineal regression of the density in function of the temperature was possible to predict the thermal expansion coefficient for two cultivates. With relationship to the viscosity, through the analysis of lineal regression, it was possible to predict the activation energy for each cultivate. For cultivates BRS-149 Nordestina and BRS-188 Paraguaçu, the thermal expansion coefficients were same, respectively, o $5.2745 \times 10^{-4}{ }^{\circ} \mathrm{C}^{-1}$ and $5.2705 \times 10^{-4}{ }^{\circ} \mathrm{C}^{-1}$, while the activation

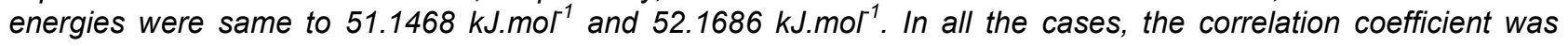
superior to 0.99 .

KEYWORDS: Expansion. Activation energy. Crude castor oil.

\section{INTRODUÇÃO}

O Brasil é o segundo exportador mundial de óleo de mamona (Ricinus communis L.), com um consumo interno relativamente pequeno (entre 10 e 15 mil toneladas/ano) e o quinto maior produtor de mamona (BRANDÃO et al., 2007). De acordo com Pina et al. (2005), no Brasil há um déficit anual de óleo de 
mamona superior a 80 mil toneladas, demanda que é satisfeita pela importação de óleo bruto proveniente da Índia e da China.

O óleo de mamona é considerado um poliéster formado por três moléculas do ácido ricinoléico (IGNÁCIO et al., 1997).

Costa et al. (2004) comentam sobre a composição do óleo de mamona em termos de ácidos graxos, em que se destaca o ácido ricinoléico (80,5\%). Outros ácidos graxos encontrados no óleo de mamona são: linoléico $(4,2 \%)$, oléico $(3,0 \%)$, palmítico $(1,0 \%)$, esteárico (1,0\%), 9,10-diidróxi-esteárico $(0,7 \%)$, eicosanóico $(0,3 \%)$ e linolênico $(0,3 \%)$.

Segundo Oliveira (2001), o processo de refino de óleos vegetais promove a remoção de:

- substâncias coloidais (gomas, proteínas, fosfatídeos e produtos de sua composição);

- ácidos graxos livres e seus sais, ácidos graxos oxidados, lactonas, acetais e polímeros;

- pigmentos (clorofila e carotenóides);

- substâncias voláteis (hidrocarbonetos, álcoois, aldeídos, cetonas e ésteres de baixo peso molecular);

- componentes inorgânicos (sais de cálcio e de outros metais, silicatos, fosfatos e outros);

- água.

Ferrari (2001) comenta que no processo de refino de óleos vegetais ocorre também a remoção de fosfolipídios, cerídeos, materiais insaponificáveis e vitaminas lipossolúveis.

A mamona cultivar BRS-149 Nordestina originou-se da linhagem CNPA M. 90-210 que, por sua vez, descende da variedade Baianita, cujas plantas têm altura média de $1,90 \mathrm{~m}$, caule de coloração verde, com cera, racemo cônico, frutos semideiscentes e sementes de coloração preta. O período entre a emergência da plântula e a floração do primeiro racemo é de 50 dias em média; o peso de 100 sementes é de $68 \mathrm{~g}$. O teor de óleo na semente é de $48,90 \%$ e a produtividade média, em semente, sem adubação, é de $1500 \mathrm{~kg} \mathrm{ha}^{-1}$ nas condições semiáridas do Nordeste Brasileiro, em anos normais, em relação à precipitação pluvial. O ciclo, a partir da emergência das plântulas à última colheita, é de 250 dias em média (COSTA, 2006).

Já a mamona cultivar BRS-188 Paraguaçu foi obtida através da seleção massal realizada na variedade local, Sangue de Boi, que originou a linhagem CNPA M. SM4, com altura média de $1,60 \mathrm{~m}$, caule de coloração roxa, com cera, racemo oval, frutos semideiscentes e sementes de coloração preta. A floração do primeiro racemo ocorre aos 54 dias, em média; o peso médio de 100 sementes é de $71 \mathrm{~g}$. O teor médio de óleo é de $47,7 \%$ e a produtividade média, sem adubação, é de 1500 kg.ha-1, nas condições semiáridas do Nordeste Brasileiro, em ano de precipitação pluvial normal (COSTA, 2006).

Segundo Chung et al. (1996), a densidade é uma das mais fundamentais propriedades de um material e está intrinsicamente relacionada com outras propriedades termofísicas. Uma dessas propriedades é o coeficiente de expansão térmica $(\beta)$.

O coeficiente de expansão térmica está relacionado com a resposta ou reação dos materiais à aplicação de calor (CALLISTER, 2002). É uma propriedade termodinâmica que fornece uma medida da variação da densidade em resposta a uma mudança na temperatura, sob uma condição de pressão constante, sendo utilizada quando se trata de convecção livre ou natural, que influencia, por exemplo, a transferência de calor em tubulações (INCROPERA; DEWITT, 1998).

No caso de materiais líquidos, o conhecimento do coeficiente de expansão térmica permite o estudo da dilatação volumétrica, ou seja, indica o quanto que o 
volume varia em virtude da variação da temperatura (CARRON; GUIMARÃES, 1997).

De acordo com Sonntag et al. (1998), o coeficiente de expansão térmica (ou coeficiente de expansão volumétrica) indica a variação de volume (V) provocada pela variação da temperatura $(\mathrm{T})$, quando a pressão $(P)$ permanece constante, conforme a equação 1.

$$
\beta=(1 / \mathrm{V}) \cdot \frac{\partial \mathrm{V}}{\partial T}
$$

Considerando a pressão constante, a equação 1 pode ser reescrita na forma de:

$$
\ln \left(\mathrm{V} / \mathrm{V}_{0}\right)=\beta .\left(\mathrm{T}-\mathrm{T}_{0}\right)
$$

Sendo $\mathrm{V}$ e $\mathrm{V}_{0}$ correspondentes ao volume final e ao volume inicial, respectivamente e; $T$ e $T_{0}$, à temperatura final e a temperatura inicial, respectivamente.

A densidade de um material é definida como sendo a razão da massa desse material pelo volume que ocupa (LEWIS, 1993). Dessa forma, considerando que a massa permanece constante entre os estados final $e$ inicial, a razão $\left(\mathrm{V} / \mathrm{V}_{0}\right)$ equivale a:

$$
\left(\mathrm{V} / \mathrm{N}_{0}\right)=\left(\mathrm{d}_{0} / \mathrm{d}\right)
$$

Sendo $d$ e $d_{0}$ correspondentes a densidade final e a densidade inicial, respectivamente.

Assim, a equação 2 pode ser reescrita na forma de:

$$
\ln \left(\mathrm{d}_{0} / \mathrm{d}\right)=\beta \cdot\left(\mathrm{T}-\mathrm{T}_{0}\right)
$$

A equação 4 relaciona a densidade de um fluido com a variação da temperatura e o coeficiente de expansão térmica (CANCIAM, 2005).
Segundo Canciam (2010), a equação 4 corresponde a uma função afim e dessa forma, o gráfico de $\ln \left(\mathbf{d}_{0} / \mathbf{d}\right)$ versus ( $\left.\mathbf{T}-\mathbf{T}_{0}\right)$ fornece uma reta, em que o coeficiente angular dessa reta corresponde, numericamente, ao coeficiente de expansão térmica ( $\beta$ ).

Padilha (1997) comenta que entre o coeficiente de expansão térmica $(\beta)$ e a energia de ligação química entre os átomos existe uma boa correlação. Materiais em que as ligações químicas são fortes apresentam um coeficiente de expansão térmica baixo. Isto porque durante o aquecimento, a frequência e a amplitude da vibração dos átomos do material aumentam. Como a dilatação térmica está associada à variação assimétrica da energia (ou força) de ligação com a distância entre os átomos e como as forças de repulsão são sempre maiores que as de atração, a distância média entre os átomos também aumenta.

A viscosidade de um fluido ( $\eta$ ) mede a resistência interna oferecida ao movimento relativo das diferentes partes desse fluido (resistência ao fluxo) (SHAMES, 1999).

Dessa maneira, a viscosidade é uma propriedade de transporte fundamental para o projeto de processos nas indústrias petroquímicas, de petróleo e químicas, em que envolvem o transporte de fluidos, mistura, agitação, filtração, concentração e transferência de calor (LIN et al., 2007). Shames (1999) complementa que é importante conhecer e controlar a viscosidade na formulação e preparação de emulsões, cremes, géis, soluções, entre outros.

Pesquisas evidenciam a influência da temperatura nos parâmetros reológicos, de maneira que, a equação do tipo Arrhenius geralmente é utilizada para descrever o efeito da temperatura na viscosidade (GRATÃO et al., 2004).

Para Mafart e Béliar (1994), a equação do tipo Arrhenius (indicada na equação 5) é expressa em termos da temperatura absoluta $(T)$, da constante universal dos gases ideais (R), da energia de ativação 
$\left(E_{a}\right)$, da viscosidade $(\eta)$ e da constante $\left(\eta_{0}\right)$, que para Pereira et al. (2003) é chamada de parâmetro de ajuste.

$$
\eta=\eta_{0} \cdot \exp \left(E_{a} / R \cdot T\right)
$$

Quanto ao parâmetro de ajuste $\eta_{0}$, Cantor (2008) considera que esse parâmetro consiste nos valores da viscosidade a altas temperaturas.

Os mesmos autores comentam que a equação do tipo Arrhenius é adequada para fluidos puros, misturas de fluidos e soluções aquosas. Entretanto, a equação é falha ao representar dados experimentais a altas temperaturas e concentrações.

Gratão et al. (2004) propõem escrever a equação do tipo Arrhenius na forma de:

$$
\ln \eta=\ln \eta_{0}+\left(E_{a} / R\right) \cdot(1 / T)
$$

A equação 6 indica a dependência da viscosidade dos fluidos com a temperatura. Pode ser utilizada, com dados experimentais de viscosidade em função da temperatura, na determinação dos parâmetros $E_{a}$ e $\mu_{0}$ (CANCIAM, 2007).

Por se tratar de uma função afim, o gráfico de Inn versus $1 / T$ fornece uma reta, em que o coeficiente angular corresponde à razão $E_{a} / R$ e o coeficiente linear, ao valor de $I n \eta_{0}$ (CANCIAM, 2007).

$O$ conhecimento da energia de ativação $\left(E_{a}\right)$ e do parâmetro de ajuste $\left(\eta_{0}\right)$ permite modelar matematicamente o comportamento reológico do fluido, bem como verificar os efeitos da temperatura sobre a sua viscosidade (GRATÃO et al., 2004).

Segundo Pereira et al. (2003), a energia de ativação $\left(E_{a}\right)$ corresponde à energia necessária para fazer um fluido se deslocar (fluir) e relaciona a sensibilidade da viscosidade devido à mudança de temperatura, onde maiores valores da energia de ativação significam que a viscosidade é relativamente mais sensivel à mudança de temperatura.

Para Ribeiro et al. (2005), as equações mais utilizadas no cálculo da viscosidade baseiam-se na Teoria do Estado Ativado de Eyring. Essa teoria considera que o fluido é formado por moléculas intercaladas por posições desocupadas (vacâncias), e estas moléculas, mesmo com o fluido em repouso, movemse a fim de ocuparem as vacâncias adjacentes a elas. A viscosidade está relacionada com a força que tende a se opor a esse movimento, sendo uma medida da friç̧ão interna do fluido. Assim, quanto maior a viscosidade, maior será a barreira potencial que uma molécula terá que vencer a fim de "saltar" para uma vacância adjacente. Essa barreira potencial é conhecida como energia de ativação.

Os objetivos deste trabalho foram predizer o coeficiente de expansão térmica $(\beta)$ a partir da análise de regressão linear de dados experimentais da densidade em função da temperatura e predizer a energia de ativação $\left(E_{a}\right)$ a partir da análise de regressão linear de dados experimentais da viscosidade em função da temperatura para o óleo bruto de mamona das cultivares BRS-149 Nordestina e BRS-188 Paragaçu.

\section{Metodologia}

A Tabela 1 relaciona os dados experimentais da densidade do óleo bruto de mamona cultivar BRS-149 Nordestina em função da temperatura. 
Tabela 1

EFEITO DA TEMPERATURA SOBRE A DENSIDADE DO ÓLEO BRUTO DE MAMONA CULTIVAR BRS-149 NORDESTINA

\begin{tabular}{c|c}
\hline Temperatura $\left({ }^{\circ} \mathrm{C}\right)$ & Densidade $\left(\mathrm{g} \cdot \mathrm{cm}^{-3}\right)$ \\
\hline-15 & 0,978 \\
-10 & 0,975 \\
-5 & 0,973 \\
0 & 0,970 \\
20 & 0,960 \\
40 & 0,950 \\
60 & 0,940 \\
80 & 0,930 \\
\hline
\end{tabular}

Fonte: Adaptado de COSTA, 2006.

A Tabela 2 relaciona os dados experimentais da densidade do óleo bruto de mamona cultivar BRS-188 Paraguaçu em função da temperatura.

\section{Tabela 2}

EFEITO DA TEMPERATURA SOBRE A DENSIDADE DO ÓLEO BRUTO DE MAMONA CULTIVAR BRS-188 PARAGUAÇU

\begin{tabular}{c|c}
\hline Temperatura $\left({ }^{\circ} \mathrm{C}\right)$ & Densidade $\left(\mathrm{g} \cdot \mathrm{cm}^{-3}\right)$ \\
\hline-15 & 0,979 \\
-10 & 0,976 \\
-5 & 0,974 \\
0 & 0,971 \\
20 & 0,962 \\
40 & 0,951 \\
60 & 0,941 \\
80 & 0,931 \\
\hline
\end{tabular}

Fonte: Adaptado de COSTA, 2006.

Com base nos dados experimentais indicados nas Tabelas 1 e 2, o coeficiente de expansão térmica $(\beta)$ do óleo bruto de mamona para cada cultivar foi obtido a partir da análise de regressão linear para os valores de $\ln \left(d_{0} / d\right)$ em função de $\left(T-T_{0}\right)$, conforme indicado pela equação 7. A equação 7 foi adaptada de Triola (1999).

$$
\begin{gathered}
\beta=\left\{\mathrm{n} \cdot\left\{\Sigma\left[\left(\mathrm{T}-\mathrm{T}_{0}\right) \cdot \ln \left(\mathrm{d}_{0} / \mathrm{d}\right)\right]\right\}-\left[\Sigma\left(\mathrm{T}-\mathrm{T}_{0}\right)\right] \cdot\left[\Sigma \ln \left(\mathrm{d}_{0} / \mathrm{d}\right)\right]\right\} / \\
\left\{\text { n. }\left[\Sigma\left(\mathrm{T}-\mathrm{T}_{0}\right)^{2}\right]-\left[\Sigma\left(\mathrm{T}-\mathrm{T}_{0}\right)\right]^{2}\right\}
\end{gathered}
$$

Nos cálculos, considerou-se como $\mathrm{T}_{0}$ a temperatura de $-15^{\circ} \mathrm{C}$ e a densidade $d_{0}$, a densidade do óleo bruto de mamona nessa temperatura. A variável $\mathbf{n}$ corresponde ao número de dados emparelhados, que nas Tabelas 1 e 2 equivale a 8 .

A Tabela 3 relaciona os dados experimentais da viscosidade $(\eta)$ do óleo bruto de mamona cultivar BRS-149 Nordestina em função da temperatura, adaptados de Costa (2006).

\section{Tabela 3}

EFEITO DA TEMPERATURA SOBRE A VISCOSIDADE DO ÓLEO BRUTO DE MAMONA CULTIVAR BRS-149 NORDESTINA

\begin{tabular}{cc}
\hline $\begin{array}{c}\text { Temperatura } \\
(\mathrm{K})\end{array}$ & $\begin{array}{c}\text { Viscosidade } \\
(\text { Pa.s })\end{array}$ \\
\hline 258,15 & 16,5490 \\
263,15 & 11,4220 \\
268,15 & 8,9640 \\
273,15 & 4,1550 \\
293,15 & 1,0816 \\
313,15 & 0,2102 \\
333,15 & 0,0929 \\
353,15 & 0,0298 \\
\hline Fonte: Adaptado de COSTA, 2006.
\end{tabular}

A Tabela 4 relaciona os dados experimentais da viscosidade ( $\eta$ ) do óleo bruto de mamona cultivar BRS-188 Paraguaçu em função da temperatura.

e-xacta, Belo Horizonte, v. 4, n. 3, p. 7-18. (2011). Editora UniBH. Disponível em: www.unibh.br/revistas/exacta/ 
De acordo com a equação (6) e com base nos dados experimentais indicados nas Tabelas 3 e 4 , os valores da razão $E_{a} / R$ e $I n \eta_{0}$ para cada cultivar foram obtidos a partir da análise de regressão linear para valores de Inn em função de (1/T), conforme indicados pelas equações 9 e 10.

\section{Tabela 4}

EFEITO DA TEMPERATURA SOBRE A VISCOSIDADE DO ÓLEO BRUTO DE MAMONA CULTIVAR BRS-188 PARAGUAÇU

\begin{tabular}{cc}
\hline $\begin{array}{c}\text { Temperatura } \\
(\mathrm{K})\end{array}$ & $\begin{array}{c}\text { Viscosidade } \\
(\text { Pa.s })\end{array}$ \\
\hline 258,15 & 16,3740 \\
263,15 & 9,5320 \\
268,15 & 8,0630 \\
273,15 & 4,0860 \\
293,15 & 0,9920 \\
313,15 & 0,1951 \\
333,15 & 0,0673 \\
353,15 & 0,0263 \\
\hline
\end{tabular}

Fonte: Adaptado de COSTA, 2006.

As equações 9 e 10 determinam, respectivamente, os valores de $E_{a} / R$ e $I n \eta_{0}$ e foram adaptadas de Triola (1999).

$$
\begin{aligned}
& E_{a} / R=\{n \cdot[\Sigma(1 / T) \cdot \ln n]-[\Sigma(1 / T) \cdot(\Sigma \ln \eta)]\} /\left\{n \cdot\left[\Sigma(1 / T)^{2}\right]-\right. \\
& \left.[\Sigma(1 / T)]^{2}\right\}
\end{aligned}
$$

$$
\operatorname{lnn} n_{0}=\left\{\Sigma \operatorname{lnn}-\left(E_{a} / R\right) \cdot \Sigma(1 / T)\right\} / n
$$

A equação 11 determina o valor do coeficiente de correlação linear $(\mathbf{r})$ para a análise de regressão linear dos dados emparelhados de $\ln \left(\mathrm{d}_{0} / \mathrm{d}\right)$ e $\left(T-\mathrm{T}_{0}\right)$. Enquanto que a equação 12 determina o valor do coeficiente de correlação linear para a análise de regressão linear dos dados emparelhados de Inn e (1/T). As equações 11 e 12 foram adaptadas de Triola (1999).

$$
\begin{gathered}
r=\left\{\mathrm{n} \cdot \Sigma\left\{\left(\mathrm{T}-\mathrm{T}_{0}\right) \cdot\left[\ln \left(\mathrm{d}_{0} / \mathrm{d}\right)\right]\right\}-\right. \\
\left.\left\{\left[\Sigma\left(\mathrm{T}-\mathrm{T}_{0}\right)\right] \cdot\left[\Sigma \ln \left(\mathrm{d}_{0} / \mathrm{d}\right)\right]\right\}\right\} /\left\{\left\{\left[\mathrm{n} \cdot \Sigma\left(\mathrm{T}-\mathrm{T}_{0}\right)^{2}\right]-\left[\Sigma\left(\mathrm{T}-\mathrm{T}_{0}\right)\right]^{2}\right\}^{1 / 2}\right. \\
\left.\left\{\left[\mathrm{n} \cdot \Sigma\left[\ln \left(\mathrm{d}_{0} / \mathrm{d}\right)\right]^{2}\right]-\left[\Sigma \ln \left(\mathrm{d}_{0} / \mathrm{d}\right)\right]^{2}\right\}^{1 / 2}\right\} \\
(11) \\
r=\left\{\mathrm{n} \cdot \Sigma\{(1 / \mathrm{T}) \cdot[\ln \eta]\}-\{[\Sigma(1 / \mathrm{T}) \cdot[\Sigma \ln \eta]\}\} /\left\{\left\{\left[\mathrm{n} \cdot \Sigma(1 / \mathrm{T})^{2}\right]-\right.\right.\right. \\
\left.\left.[\Sigma(1 / \mathrm{T})]^{2}\right\}^{1 / 2} \cdot\left\{\left[\mathrm{n} \cdot \Sigma[\ln \eta]^{2}\right]-[\Sigma \ln n]^{2}\right\}^{1 / 2}\right\}
\end{gathered}
$$

\section{Resultados e Discussão}

A FIG. 1 ilustra o gráfico de $\ln \left(\mathbf{d}_{0} / \mathbf{d}\right)$ versus $\left(\mathbf{T}-\mathbf{T}_{0}\right)$ para os dados do óleo bruto de mamona cultivar BRS-149 Nordestina, indicados na Tabela 1.

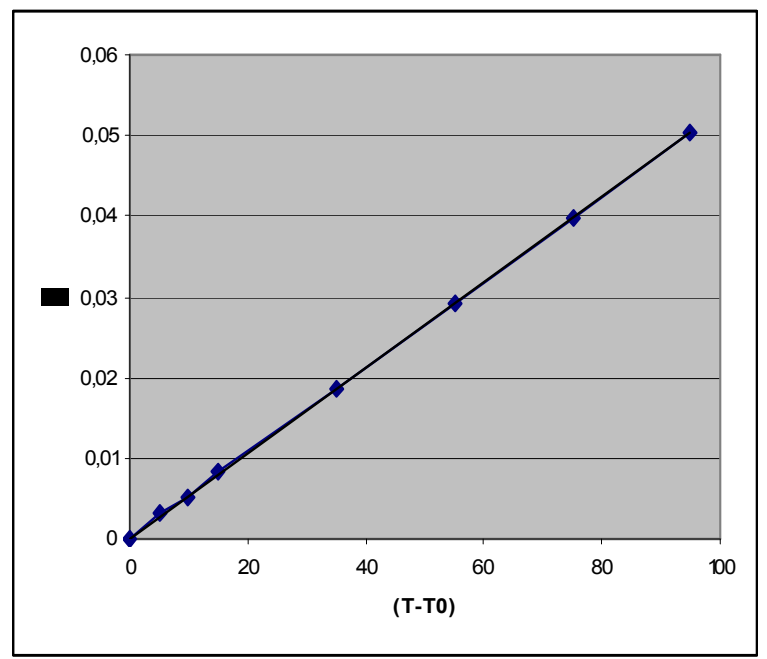

Figura 1. Gráfico $\ln \left(\mathrm{d}_{0} / \mathrm{d}\right)$ versus $\left(T-\mathrm{T}_{0}\right)$ para o óleo bruto de mamona cultivar BRS-149 Nordestina Considerando a FIG. 1, a equação para a reta obtida nessa figura equivale a:

$$
\ln \left(d_{0} / d\right)=5,2745 \times 10^{-4} \cdot\left(T-T_{0}\right)+1,2877 \times 10^{-4}
$$


Com base na equação 11 , o coeficiente de correlação (r) para o gráfico da FIG. 1 é igual a 0,9999.

A FIG. 2 ilustra o gráfico de $\ln \left(\mathbf{d}_{0} / \mathrm{d}\right)$ versus $\left(\mathbf{T}-\mathrm{T}_{0}\right)$ para os dados do óleo bruto de mamona cultivar BRS-188 Paraguaçu, indicados na Tabela 2.

A equação da reta obtida no gráfico ilustrado na FIG. 2 equivale a:

$$
\ln \left(\mathrm{d}_{0} / \mathrm{d}\right)=5,2705 \times 10^{-4} .\left(\mathrm{T}-\mathrm{T}_{0}\right)-6,6267 \times 10^{-6}
$$



Figura 2. Gráfico $\ln \left(\mathrm{d}_{0} / \mathrm{d}\right)$ versus $\left(\mathrm{T}-\mathrm{T}_{0}\right)$ para o óleo bruto de mamona cultivar BRS-188 Paraguaçu

O coeficiente de correlação (r) para o gráfico da FIG. 2, com base na equação 11, é igual a 0,9998.

Dessa forma, com base na equação 4 , os coeficientes de expansão térmica $(\beta)$ dos óleos brutos de mamona cultivares BRS-149 Nordestina e BRS-188 Paraguaçu correspondem, respectivamente a, 5,2745 $\times 10^{-4}{ }^{\circ} \mathrm{C}^{-1} \mathrm{e}$ $5,2705 \times 10^{-4}{ }^{\circ} \mathrm{C}^{-1}$.

Pode-se observar que os coeficientes de expansão térmica para as cultivares são bastante próximos.

Em pesquisas na literatura, constatou-se a ausência de dados experimentais do coeficiente de expansão térmica para o óleo bruto de mamona.
A Tabela 5 relaciona alguns valores do coeficiente de expansão térmica para óleos vegetais refinados encontrados na literatura.

Nos trabalhos citados na Tabela 5, pode-se observar a proximidade nos coeficientes de expansão térmica para os diferentes óleos vegetais refinados, na ordem de $7,0 \times 10^{-4}{ }^{\circ} \mathrm{C}^{-1}$.

Os óleos brutos de mamona das cultivares Nordestina e Paraguaçu apresentaram coeficientes de expansão térmica na ordem de $5,2 \times 10^{-4} \circ \mathrm{C}^{-1}$, ou seja, em relação aos óleos vegetais refinados listados na Tabela 5, apresentaram uma diferença na ordem de $1,8 \times 10^{-4} \circ \mathrm{C}^{-1}$. Isto se deve provavelmente, devido à diferença de composição em termos de alguns constituintes, em virtude do processo de refino.

\section{Tabela 5}

VALORES DO COEFICIENTE DE EXPANSÃO TÉRMICA PARA ÓLEOS VEGETAIS ENCONTRADOS NA LITERATURA

\begin{tabular}{ccc}
$\begin{array}{c}\text { Óleo vegetal } \\
\text { refinado }\end{array}$ & $\begin{array}{c}\text { Coeficiente de } \\
\text { Expansão } \\
\text { Térmica }\left({ }^{\circ} \mathrm{C}^{-1}\right)\end{array}$ & Referência \\
\hline Azeite de oliva & $7,20 \times 10^{-4}$ & $\begin{array}{c}\text { Cabral e Lago } \\
(2002)\end{array}$ \\
$\begin{array}{c}\text { Óleo de } \\
\text { algodão }\end{array}$ & $7,4355 \times 10^{-4}$ & Canciam (2005) \\
$\begin{array}{c}\text { Óleo de soja } \\
\text { Óleo de } \\
\text { gergelim }\end{array}$ & $7,4676 \times 10^{-4}$ & Canciam (2008) \\
& $7,46 \times 10^{-4}$ & Canciam (2010)
\end{tabular}

Outra possibilidade que justifique a diferença de valores do coeficiente de expansão térmica está associada à composição em termos dos ácidos graxos.

Segundo Padilha (1997), materiais em que as ligações químicas são fortes apresentam um coeficiente de expansão térmica baixo.

e-xacta, Belo Horizonte, v. 4, n. 3, p. 7-18. (2011). Editora UniBH. Disponível em: www.unibh.br/revistas/exacta/ 
De acordo com Costa (2006), o óleo de mamona é um óleo vegetal que se diferencia dos demais óleos vegetais pela grande quantidade de grupos hidroxila, encontrados no ácido ricinoléico (componente de maior concentração no óleo de mamona). Os grupos hidroxila conferem ao triglicerídeo do ácido ricinoléico certa estabilidade em larga faixa de temperaturas. Essa certa estabilidade é decorrente da formação de pontes de hidrogênio entre as moléculas.

As pontes de hidrogênio são ligações intermoleculares fortes (FONSECA, 1992).

Dessa forma, provavelmente em virtude dessas pontes de hidrogênio formadas, o coeficiente de expansão térmica do óleo bruto de mamona é menor em relação aos outros óleos vegetais.

Carron e Guimarães (1997) comentam que no caso dos materiais líquidos, o conhecimento do coeficiente de expansão térmica permite o estudo da dilatação volumétrica, ou seja, indica o quanto que o volume varia em virtude da variação da temperatura. Nesse sentido, considerando, por exemplo, para um aumento de temperatura de $10^{\circ} \mathrm{C}$, o aumento no volume de óleo refinado de algodão é de aproximadamente 7,4 litros para cada mil litros desse óleo. No presente trabalho, pode-se observar que com um aumento de temperatura de $10^{\circ} \mathrm{C}$, o aumento no volume de óleo bruto de mamona é de aproximadamente 5,3 litros para cada mil litros, independente da cultivar considerada.

A FIG. 3 ilustra o gráfico de Inn versus (1/T) para os dados do óleo bruto de mamona cultivar BRS-149 Nordestina, indicados na Tabela 3.

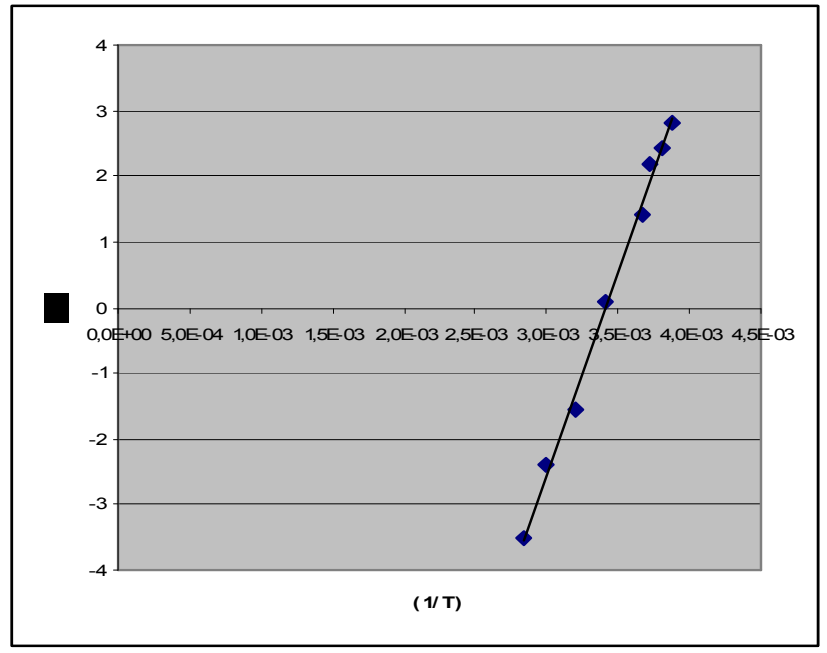

Figura 3. Gráfico Inn versus (1/T) para o óleo bruto de mamona cultivar BRS-149 Nordestina

Considerando a FIG. 3, a equação da reta obtida nessa figura equivale a:

$$
\ln \eta=-20,9626+6151,8906 .(1 / T)
$$

O coeficiente de correlação (r), para este caso, com base na equação 12 , é igual a 0,9982 .

Com base em Gratão et al. (2004), a equação 15 corresponde ao modelo matemático que descreve o comportamento reológico para o óleo bruto de mamona cultivar BRS-149 Nordestina.

A FIG. 4 ilustra o gráfico de Inn versus (1/T) para os dados do óleo bruto de mamona cultivar BRS-188 Paraguaçu, indicados na Tabela 4. 


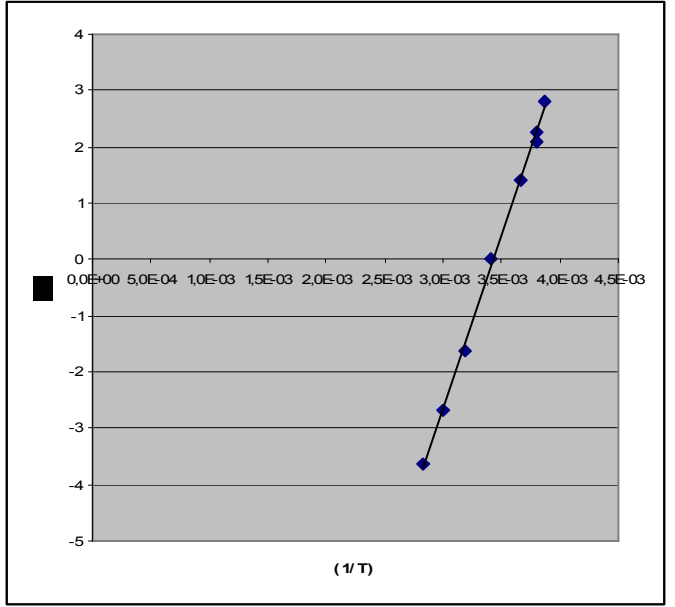

Figura 4. Gráfico In $\mu$ versus (1/T) para o óleo bruto de mamona cultivar BRS-188 Paraguaçu

A equação para a reta obtida no gráfico ilustrado na FIG. 4 equivale a:

$$
\ln \eta=-21,5004+6274,7942 \cdot(1 / T)
$$

O coeficiente de correlação (r), para este caso, com base na equação 12 , é igual a 0,9989 .

Com base em Gratão et al. (2004), a equação 16 corresponde ao modelo matemático que descreve o comportamento reológico para o óleo bruto de mamona cultivar BRS-188 Paraguaçu.

Dessa forma, os parâmetros de ajuste $\left(\eta_{0}\right)$ correspondem, respectivamente, para as cultivares BRS-149 Nordestina e BRS-188 Paraguaçu, os valores de $7,8715 \times 10^{-10}$ Pa.s e 4,5972 × $10^{-10}$ Pa.s.

Considerando que a constante universal dos gases ideais $(R)$ é igual a $8,314 \times 10^{-3} \mathrm{~kJ} \mathrm{~mol}^{-1} \cdot \mathrm{K}^{-1}$, os valores para a energia de ativação $\left(E_{a}\right)$ correspondem, respectivamente, para as cultivares BRS-149 Nordestina e BRS-188 Paraguaçu, 51,1468 kJ.mol ${ }^{-1}$ e $52,1686 \mathrm{~kJ}^{\mathrm{mol}}{ }^{-1}$.

Com relação à energia de ativação $\left(E_{a}\right)$, a literatura não dispõe de dados experimentais para esta grandeza, considerando o óleo bruto de mamona.
Também não são encontrados valores de energia de ativação para outros óleos vegetais, o que dificulta a comparação.

Gratão et al. (2004) determinaram como valores da energia de ativação $\left(E_{a}\right)$ para soluções aquosas de açúcar invertido com $59,68 \%$ e $89,88 \%$ de inversão, entre 17 e $65^{\circ} \mathrm{C}$, respectivamente, $(70,5 \pm 0,4) \mathrm{kJ}_{\mathrm{mol}}{ }^{-1}$ e $(86,5 \pm 3,6) \mathrm{kJ} \cdot \mathrm{mol}^{-1}$. Todos com um coeficiente de correlação igual a 0,99.

Canciam (2007) determinou como valores da energia de ativação $\left(E_{a}\right)$ para soluções aquosas contendo 20 , 40 e $60 \%$ (em massa) de sacarose, entre 10 e $85^{\circ} \mathrm{C}$,

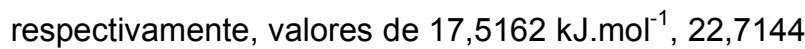
$\mathrm{kJ} \cdot \mathrm{mol}^{-1}$ e $34,8019 \mathrm{~kJ} \cdot \mathrm{mol}^{-1}$.

Dessa forma, pode-se observar que os valores encontrados no presente trabalho, a energia de ativação dos óleos brutos de mamona, independente das cultivares, encontram-se situados entre os valores da energia de ativação para as soluções aquosas de sacarose e soluções aquosas de açúcar invertido.

Costa (2006) comenta que a presença de grupos hidroxila no triglicerídeo do ácido ricinoléico confere ao óleo de mamona, além de uma maior densidade em relação a outros óleos vegetais, uma maior viscosidade.

Segundo Ribeiro et al. (2005), pela Teoria do Estado Ativado de Eyring, quanto maior for a viscosidade, maior será a barreira potencial (energia de ativação) que uma molécula terá que vencer a fim de "saltar" para uma vacância adjacente.

Por sua vez, maiores valores da energia de ativação significam que a viscosidade é relativamente mais sensível à mudança de temperatura (PEREIRA et al., 2003).

Dessa forma, sugere-se que a energia de ativação $\left(E_{a}\right)$ para os óleos brutos de mamona, independente das cultivares, é considerada "alta", implicando que a sua viscosidade é relativamente mais sensível à 
mudança de temperatura, ou seja, pequenas variações de temperatura são necessárias para modificar rapidamente a viscosidade do óleo bruto de mamona, independente da cultivar.

Com relação à temperatura, a própria equação do tipo Arrhenius (equação 5) indica a dependência da viscosidade com a temperatura, de maneira que o aumento da temperatura provoca uma diminuição da viscosidade. Esta redução de viscosidade com o aumento da temperatura é atribuído, segundo Grangeiro et al. (2007), ao aumento das distâncias intermoleculares provocadas pelo aquecimento. $\mathrm{O}$ aumento das distâncias reduz as forças atrativas intermoleculares, diminuindo a viscosidade.

\section{ConClusõEs}

$\mathrm{Na}$ faixa de temperatura de -15 e $80^{\circ} \mathrm{C}$, os coeficientes de expansão térmica para as cultivares BRS-149 Nordestina e BRS-188 Paraguaçu foram próximos.

Em relação a outros óleos vegetais refinados, o coeficiente de expansão térmica para o óleo bruto de mamona foi menor, provavelmente, em virtude das pontes de hidrogênio formadas entre os grupos hidroxila presentes no triglicerídeo do ácido ricinoléico e em decorrência da diferença de composição entre óleos vegetais refinados e brutos.

Para a mesma faixa de temperatura, a energia de ativação da cultivar BRS-188 Paraguaçu foi um pouco maior em relação a cultivar BRS-149 Nordestina.

Em virtude da presença de grupos hidroxila presentes no triglicerídeo do ácido ricinoléico, sugere-se que o valor da energia de ativação é "alto" e por isso, pequenas variações de temperatura são necessárias para modificar rapidamente a sua viscosidade.
Devido à diversa aplicação do óleo bruto de mamona e à escassez de dados experimentais disponíveis na literatura, o conhecimento do efeito da temperatura na viscosidade e na dilatação volumétrica torna-se indispensável, principalmente nos cálculos de engenharia que envolvem a seleção de equipamentos e o dimensionamento de bombas e tubulações, assim como para a implementação de um efetivo controle de processos.

\section{NOMENCLATURA}

$d$ = densidade na temperatura final;

$\mathrm{d}_{0}=$ densidade na temperatura inicial;

$\exp =$ exponencial;

$\mathrm{E}_{\mathrm{a}}=$ energia de ativação;

In = logaritmo neperiano;

$\mathrm{n}$ = número de dados amostrais emparelhados;

$\mathrm{P}=$ pressão;

$\mathrm{R}=$ constante universal dos gases ideais;

$r=$ coeficiente de correlação linear;

$\mathrm{T}=$ temperatura final;

$\mathrm{T}_{0}=$ temperatura inicial;

$V=$ volume final;

$\mathrm{V}_{0}=$ volume inicial;

$\beta=$ coeficiente de expansão térmica;

$\partial=$ derivada parcial;

$\eta=$ viscosidade;

$\eta_{0}=$ parâmetro de ajuste;

$\Sigma=$ somatório. 


\section{REFERÊNCIAS}

BRANDÃO, K.S.R. et al. Otimização da transesterificação etílica do óleo de mamona usando o delineamento composto central rotacional (DCCR). In: CONGRESSO DA REDE BRASILEIRA DE TECNOLOGIA DE BIODIESEL, 2., 2007, Brasília. Anais... Brasília: SETEC/MCT, 2007.

CABRAL, F.; LAGO, A. Física 2. São Paulo: Harbra, 2002, 516p.

CALLISTER, W.D. Ciência e Engenharia de Materiais: uma introdução. Rio de Janeiro: Livros Técnicos e Científicos Editora, 2002, 450p.

CANCIAM, C.A. Predição do coeficiente de expansão térmica do óleo de algodão. REVISTA PUBLICATIO UEPG - CIÊNCIAS EXATAS E DA TERRA, CIÊNCIAS AGRÁRIAS E ENGENHARIAS, v. 11, n. 3, p. 27-31, Dez. 2005.

CANCIAM, C.A. Efeito da temperatura na viscosidade de soluções de sacarose. In: SEMANA DE TECNOLOGIA EM ALIMENTOS, 5., 2007, Ponta Grossa. Anais... Ponta Grossa: UTFPR, 2007. CDROM.

CANCIAM, C.A. Predição do coeficiente de expansão térmica do óleo de soja (Glicine max). Revista Tecnológica, v. 17, p. 13-18, 2008.

CANCIAM, C.A. Predição do coeficiente de expansão térmica do óleo de gergelim (Sesamum indicum L.) através da aplicação de regressão linear. Revista exacta, v.3, n.1, 2010.

CANTOR, J.M. Progress in Food Engineering Research and Development. New York: Nova Science Publishers, 2008, 294p.

CARRON, W.; GUIMARÃES, O. As Faces da Física. São Paulo: Moderna, 1997, 672p.

CHUNG, S.K.; THIESSEN, D.B.; RHIM, D.B. A non contact measurement technique for the density and the thermal expansion coefficient of solid and liquid material. Review of Scientific Instruments, New York, v. 67, n. 9, p. 3003-3381, 1996.

COSTA, H.M. et al. Efeito do óleo de mamona em composições de borracha natural contendo sílica. Polímeros: Ciência e Tecnologia, São Carlos, v. 14, n. 1, p. 46-50, Jan./Marc. 2004.

COSTA, T.L. Características físicas e físico-químicas do óleo de duas cultivares de mamona. Dissertação de Mestrado em Engenharia Agrícola - Universidade
Federal de Campina Grande, Campina Grande, 2006, $113 p$.

FERRARI, R.A. Componentes minoritários de óleos vegetais. Revista Óleos \& Grãos, ano IX, n. 58, p. 20-28, Jan./Fev. 2001.

FONSECA, M.R.M. Química Geral. São Paulo: Editora FTD, 1992, 400p.

GRANGEIRO, A.A. et al. Viscosidades de polpas concentradas de figo-da-Índia. Revista Brasileira de Agrociência, Pelotas, v. 13, n. 2, p. 219-224, Jun. 2007.

GRATÃO, A.C.A.; BERTO, M.I.; SILVEIRA JÚNIOR, V. Reologia do açúcar líquido invertido: influência da temperatura na viscosidade. Ciência e Tecnologia de Alimentos, Campinas, v. 24, n. 4, p. 652-656, Out./Dez. 2004.

IGNÁCIO, H. et al. Uso da poliuretana derivada do óleo de mamona para preencher defeitos ósseos diafisários segmentares do rádio: estudo experimental em coelhos. Revista Brasileira de Ortopedia, São Paulo, v. 32, n. 10, p. 815-821, Out. 1997.

INCROPERA, F.P.; DEWITT, D.P. Fundamentos de transferência de calor e massa. Rio de Janeiro: Livros Técnicos e Científicos, 1998, 494p.

LEWIS, M.J. Propiedades físicas de los alimentos y de los sistemas de procesado. Zaragoza: Acribia, 1993, 494p.

LIN, C.F.; HSIEH, H.M.; LEE, L.S. Estimations of the viscosities of binary mixtures with different equations of state and mixing rules. Journal of the Chinese Institute of Chemical Engineers, Taiwan, v. 38, p. 119, 2007.

MAFART, P.; BÉLIARD, E. Ingeniería industrial alimentaria volumen II: Técnicas de separación. Zaragoza: Acribia, 1994. 277 p.

OLIVEIRA, C.G. Proposta de modelagem transiente para a clarificação de óleos vegetais - experimentos cinéticos e simulação do processo industrial. Dissertação de Mestrado em Engenharia Química Universidade Federal de Santa Catarina, Florianópolis, 2001, 144p.

PADILHA, A.F. Materiais de Engenharia: microestrutura e propriedades. São Paulo: Hemus, 1997, 349p. 
PEREIRA, E.A.; QUEIROZ, A.J.M.; FIGUEIRÊDO, R.M.F. Comportamento reológico de mel da abelha uruçu (Melípona scutellaris, L.). Revista Ciências Exatas e Naturais, Guarapuava, v. 5, n. 2, p. 179186, Jul./Dez. 2003.

PINA, M. et al. Novas alternativas de valorização para dinamizar a cultura da mamona no Brasil. Cadernos de Ciência \& Tecnologia, Brasília, v. 22, n. 2, p. 453462, Maio/Ago. 2005

RIBEIRO, L.D. et al. Cálculo de viscosidade de misturas não eletrolíticas. In: CONGRESSO BRASILEIRO DE ENGENHARIA QUÍMICA EM INICIAÇÃO CIENTÍFICA, 6., 2005, Campinas. Anais... Campinas: Unicamp, 2005.
SHAMES, I.H. Mecânica dos Fluidos - volume 1. São Paulo: Edgard Blücher, 1999. 192 p.

SONNTAG, R.E.; BORGNAKKE, C.; VAN WYLEN, G.J. Fundamentos da Termodinâmica. São Paulo: Edgard Blücher, 1998, 537p.

TRIOLA, M.F. Introdução à Estatística. Rio de Janeiro: Livros Técnicos e Científicos, 1999, 410p. 